\title{
Surface undulations of Antarctic ice streams tightly controlled by bedrock topography
}

\author{
J. De Rydt ${ }^{1,2}$, G. H. Gudmundsson ${ }^{2}$, H. F. J. Corr ${ }^{2}$, and P. Christoffersen ${ }^{1}$ \\ ${ }^{1}$ Scott Polar Research Institute, University of Cambridge, Lensfield Road, Cambridge CB2 1ER, UK \\ ${ }^{2}$ British Antarctic Survey, High Cross Madingley Road, Cambridge CB3 OET, UK
}

Correspondence to: J. De Rydt (janryd69@bas.ac.uk)

Received: 25 September 2012 - Published in The Cryosphere Discuss.: 22 October 2012

Revised: 21 January 2013 - Accepted: 22 January 2013 - Published: 1 March 2013

\begin{abstract}
Full Stokes flow-line models predict that fastflowing ice streams transmit information about their bedrock topography most efficiently to the surface for basal undulations with length scales between 1 and 20 times the mean ice thickness. This typical behaviour is independent of the precise values of the flow law and sliding law exponents, and should be universally observable. However, no experimental evidence for this important theoretical prediction has been obtained so far, hence ignoring an important test for the physical validity of current-day ice flow models. In our work we use recently acquired airborne radar data for the Rutford Ice Stream and Evans Ice Stream, and we show that the surface response of fast-flowing ice is highly sensitive to bedrock irregularities with wavelengths of several ice thicknesses. The sensitivity depends on the slip ratio, i.e. the ratio between mean basal sliding velocity and mean deformational velocity. We find that higher values of the slip ratio generally lead to a more efficient transfer, whereas the transfer is significantly dampened for ice that attains most of its surface velocity by creep. Our findings underline the importance of bedrock topography for ice stream dynamics on spatial scales up to 20 times the mean ice thickness. Our results also suggest that local variations in the flow regime and surface topography at this spatial scale cannot be explained by variations in basal slipperiness.
\end{abstract}

\section{Introduction}

The flow regime of the Antarctic ice sheet is dominated by an extensive network of fast-flowing ice streams and outlet glaciers, which account for $90 \%$ of the ice sheet's dis- charge (Bamber et al., 2000). It is clear that accurate predictions about the mass balance of the Antarctic Ice Sheet rely on a good understanding of the dynamical mechanisms that control the flow of these rapidly moving features. An important role in the regulation of ice stream flow is played by their basal conditions, i.e. bedrock topography and variations in basal slipperiness. A possible way to obtain information about the conditions at the base involves the inversion of irregularities at the surface arising from bedrock bumps or variations in slipperiness. Control method inversion uses accurate remote sensing data about the surface velocity field in combination with theoretical ice flow models to obtain information about the basal shear stress distribution of ice streams (MacAyeal et al., 1995; Vieli and Payne, 2003; Joughin et al., 2004, 2006; Arthern and Gudmundsson, 2010). In addition, statistical inversion methods have been developed to study the simultaneous retrieval (or update) of bed topography and basal slipperiness from surface topography and velocity measurements (Gudmundsson, 2004a; Raymond and Gudmundsson, 2009; Raymond-Pralong and Gudmundsson, 2011).

All inversion methods obtain information about the bed in an indirect way and rely on a good theoretical understanding of how surface properties connect to conditions at the bed. Most advances in our understanding of this relation have come from the theoretical analysis of ice dynamics equations subjected to varying boundary conditions (see e.g. Nye, 1959; Budd, 1970; Landon and Raymond, 1978, for earlier studies). A substantial part of the subsequent work has concentrated on the transfer of basal disturbances in topography and slipperiness to the surface, and how they influence quantities that are directly observable such as the surface velocity and surface topography. A systematic theoretical analysis 
of transfer amplitudes using perturbation methods was initiated by Hutter (1983), and his results were later corrected and extended by Johannesson (1992). An analytical solution for the three-dimensional full Stokes problem with a linear ice rheology and linear sliding law was obtained by Gudmundsson (2003), and subsequently studied in the shallowice-stream approximation by Gudmundsson (2008). Numerical solutions for a nonlinear flow and nonlinear sliding law were obtained by Raymond and Gudmundsson (2005).

It is often thought that variations in basal slipperiness are the principle cause of changing flow conditions along an ice stream, and the role of the bedrock topography is rarely addressed directly in this context. However, one of the predictions that emerge from the full Stokes computations in Gudmundsson (2003) is the characteristic behaviour of the bed-to-surface transfer of small-amplitude basal undulations (small with respect to the ice thickness). It is found that, for ice streams which acquire most of their speed through basal sliding, the transmission of bedrock irregularities to the surface is most efficient for length scales between 1 and 20 times the mean ice thickness, whereas variations in slipperiness only have an impact at much longer wavelengths. Theory therefore predicts that the surface topography of fast-flowing ice streams should be dominated by features of several ice thicknesses in length which reflect the presence of similar undulations at the bed. Direct observational evidence for this result has not been obtained so far, despite its importance as a test for theoretical models. In this paper, we address the issue directly for the first time using airborne radar data along the fast-flowing Rutford and Evans ice streams, as well as the nearly stagnant Carlson Inlet, West Antarctica.

The paper is organized as follows. In Sect. 2 we introduce the theoretical framework that describes the surface response of a flowing ice mass to spatial changes at its base. The remainder of the paper focuses on the experimental validation of this framework. Section 3 contains an overview of the radar data and a discussion about the methodology which was used to estimate the transfer function for each flight line. Results of our analysis are presented in Sect. 4, followed by a discussion of the main findings in Sect. 5. Conclusions are given in Sect. 6.

\section{Theoretical transfer functions}

We express the bed topography $(b)$, basal slipperiness distribution $(c)$ and surface topography $(s)$ as the sum of a mean value, henceforth denoted by a superscript (0), and deviations around the mean value:

$b(x, y)=b^{(0)}+\Delta b(x, y)$,

$(x, y)=c^{(0)}+\Delta c(x, y)$,

$s(t, x, y)=s^{(0)}+\Delta s(t, x, y)$,

where we assume that $b$ and $c$ are independent of time. The perturbative study of transfer properties of a flowing ice mass

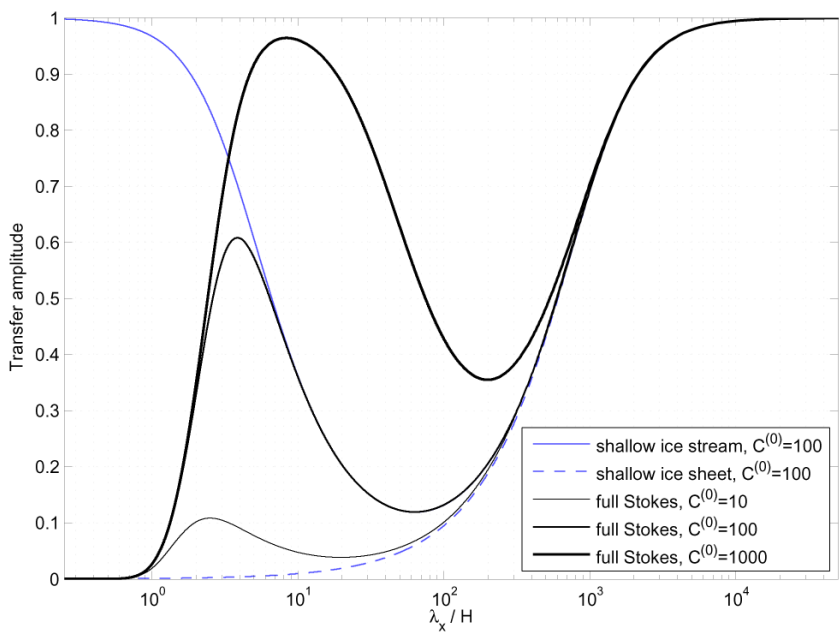

Fig. 1. Two-dimensional steady-state transfer amplitude $T_{\mathrm{sb}}\left(\lambda_{\mathrm{x}}\right)$ for $\alpha=0.003 \mathrm{rad}$ and three values of the mean slip ratio: $C^{(0)}=10$, $C^{(0)}=100$ and $C^{(0)}=1000$. Black curves represent the full Stokes solution (Gudmundsson, 2003), whereas dashed and dotted blue lines correspond to the shallow ice stream and shallow ice sheet approximation, respectively (Gudmundsson, 2008). All solutions are given for a linear ice rheology and linear sliding law.

relies on the assumption that the deviations from the mean values are small, i.e. $\Delta b / H \ll 1$ and $\Delta s / H \ll 1$ with $H$ the mean thickness of the ice, and $\Delta c / c^{(0)} \ll 1$.

It is common to choose the mean values $b^{(0)}, c^{(0)}$ and $s^{(0)}$ as the solutions for the undisturbed flow (i.e. the zeroth order solution) of an ice mass of uniform thickness $H$ along an infinite-plane-slab geometry with mean surface slope $\alpha$. We refer to Sect. 3.2 and Fig. 3 for more details about the application of this expansion to our data. If the $\mathrm{x}$-axis is taken along the upper surface in the direction of the flow and the $\mathrm{z}$-axis normal to the surface, then one finds the following expressions (for a linear ice rheology and linear sliding law):

$s^{(0)}=0$,

$b^{(0)}=-H$,

$c^{(0)}=v_{b, x}^{(0)} / \sin (\alpha) \rho g H$,

where $v_{b, x}^{(0)}$ is the mean forward basal sliding velocity, $g$ the acceleration due to gravity and $\rho$ the ice density.

It is often beneficial to introduce scalings that replace the dimensionful flow variables by dimensionless quantities. In dimensionless variables, the slipperiness distribution $c^{(0)}$ is replaced by $C^{(0)}=\eta c^{(0)} / H$, with $\eta$ the ice viscosity. This quantity is often referred to as the slip ratio, and can alternatively be written as the ratio of the mean basal sliding velocity and the forward deformational velocity at the surface:

$C^{(0)}=\frac{v_{b, x}^{(0)}}{v_{d, x}^{(0)}}, \quad$ with

$v_{d, x}^{(0)}=\sin (\alpha) \rho g H^{2} / \eta$. 
The Eqs. (2) and (3) are used to solve the momentum equations and boundary conditions of the flow, up to first order in the small perturbances. The resulting relations between the perturbances define the first-order transfer functions, $T$, which describe the transient response of a glacier's surface to small disturbances at its base:

$\Delta s=T_{\mathrm{sb}} \Delta b+T_{\mathrm{sc}} \Delta c$.

Each transfer function has two subindices: the first index relates to the effect and the second to the cause. For example, $T_{\mathrm{sb}}$ describes the effect on the surface topography caused by perturbations in the bedrock topography. The transfer functions are complex expressions; the amplitude corresponds to the amplitude ratio between the surface and basal perturbations, whereas the phase describes the shift of the surface undulations with respect to the perturbations at the bed. Our work will focus on the amplitude of the transfer function; phase shifts will not be considered as results remained inconclusive.

An analytical solution for the full Stokes system with a linear ice rheology and linear sliding law has been presented by Gudmundsson (2003); see his Eqs. (75) and (76). The solutions are most easily obtained in their Fourier-transformed form, and are written in terms of transversal $\left(\lambda_{y}\right)$ and longitudinal $\left(\lambda_{\mathrm{x}}\right)$ wavelength components. The dependence of the transfer functions on the ice viscosity and the mean ice thickness is only indirect via the mean slip ratio, whereas the mean surface slope enters the solutions as a free parameter.

In the following, we are interested in the characteristic behaviour of the transfer amplitude $T_{\mathrm{sb}}$ in the steady-state limit $(t \rightarrow \infty)$ and for long transverse wavelengths $\lambda_{\mathrm{y}}$. The latter requirement is motivated by the fact that the flow of an ice stream along a flow-line will be treated as a one-dimensional problem, and basal perturbations transverse to the flow direction will be neglected. This effectively corresponds to taking the limit $\lambda_{\mathrm{y}} \rightarrow \infty$ of the three-dimensional solution $T_{\mathrm{sb}}\left(\lambda_{\mathrm{x}}\right.$, $\left.\lambda_{\mathrm{y}}, C^{(0)}, \alpha\right)$. The flow-line approximation is graphically represented in Fig. 1 for $\alpha=0.003$ rad (a typical value encountered for ice streams) and various values of the slip ratio $C^{(0)}$.

The full Stokes solution shows three distinctive regimes: for short wavelengths $\lambda_{\mathrm{x}}<H$, the transfer is close to zero; for long wavelengths $\lambda_{\mathrm{x}}>10^{3} \mathrm{H}$, the transfer converges towards unity; and for intermediate wavelengths $H<\lambda_{\mathrm{x}}<$ $10^{3} \mathrm{H}$, the transfer function has a local maximum. The amplitude of this maximum is strongly dependent on the value of $C^{(0)}$. For low values of the slip ratio, i.e. $C^{(0)}<10$, the peak is almost absent, whereas it is much more prominent for $C^{(0)}>100$. In other words, for slippery beds - such as those underneath fast-flowing ice streams - the transfer of information about bedrock undulations to the surface is expected to happen very efficiently at intermediate wavelengths. This feature distinguishes ice streams with a high slip ratio from slower-moving ice that obtains a substantial part of its surface speed by internal creep, such as the surrounding ice sheet. It is exactly this important distinction that will be the subject of our study. The theoretical form of $T_{\mathrm{sb}}$ will be compared to its estimated value from observational data for fast as well as slow-flowing sections of the Antarctic ice sheet. Results will provide important insights into the applicability of the full Stokes theory to ice stream modelling. Commonly used approximations such as the shallow ice sheet approximation and shallow ice stream approximation fail to reproduce the transfer behaviour of the full Stokes system for wavelengths below 100 and 10 ice thicknesses, respectively (see Fig. 1 and Gudmundsson, 2008), and are therefore less appropriate as a theoretical basis for our study.

It is important to note that the solutions presented in Fig. 1 are only valid for a linear ice rheology (Glen's flow law exponent $n=1$ ) and linear sliding law (sliding law exponent $m=1$ ). Therefore, the question remains whether the predicted peak in the transfer amplitude is related to this specific choice of $n$ and/or $m$, or whether it is more general, and therefore present for more realistic values of $n>1$ and $m>1$. Since an analytical solution to the full Stokes transfer problem for arbitrary $n$ and $m$ is not known, an answer to this question relies on the numerical analysis of the momentum equations and boundary conditions. This numerical problem was addressed by Raymond and Gudmundsson (2005) in a flowline setting. The authors concluded that there is no qualitative difference between the solutions for a linear ice rheology and a non-linear medium, with only a small increase of the transfer for $n>1$, and a shift of the local maximum towards longer wavelengths. Also the dependence of $T_{\mathrm{sb}}$ on $m$ is weak, with a minor decrease in the transfer amplitude for $m>1$. In addition, it is expected that $T_{\mathrm{sb}}$ exhibits a fast convergence towards a limiting curve for increasing values of $m$. As a consequence, the transfer amplitude for a plastic bed $(m=\infty)$ is roughly equal to the transfer amplitude for $m \sim 10$. In summary, a local peak in the transfer function is to be expected for high values of the slip ratio, irrespective of the physical values of $n$ and $m$.

\section{Data and methods}

\subsection{Data description}

In this work we used unmigrated bed and surface returns from an extensive radio echo sounding survey (RES) over the southwestern margin of the Filchner-Ronne Ice Shelf and the adjacent West Antarctic ice sheet (inset of Fig. 2). In January 2007, the British Antarctic Survey flew 22 RES sorties from two remote field camps (Rutford Ice stream Camp at $78^{\circ} 08^{\prime} \mathrm{S}, 83^{\circ} 54^{\prime} \mathrm{S}$ and Ski-Blu at $74^{\circ} 51^{\prime} \mathrm{S}, 71^{\circ} 35^{\prime} \mathrm{W}$ ), covering a total distance of about $20000 \mathrm{~km}$. The radar platform consisted of a Twin Otter equipped with the ice-sounding radar system PASIN (Polarimetric Airborne Survey INstrument), dual-frequency carrier-wave GPS for navigation and high-frequency radar altimeter. The RES system operated at a center frequency of $150 \mathrm{MHz}$ and $12 \mathrm{MHz}$ bandwidth. 


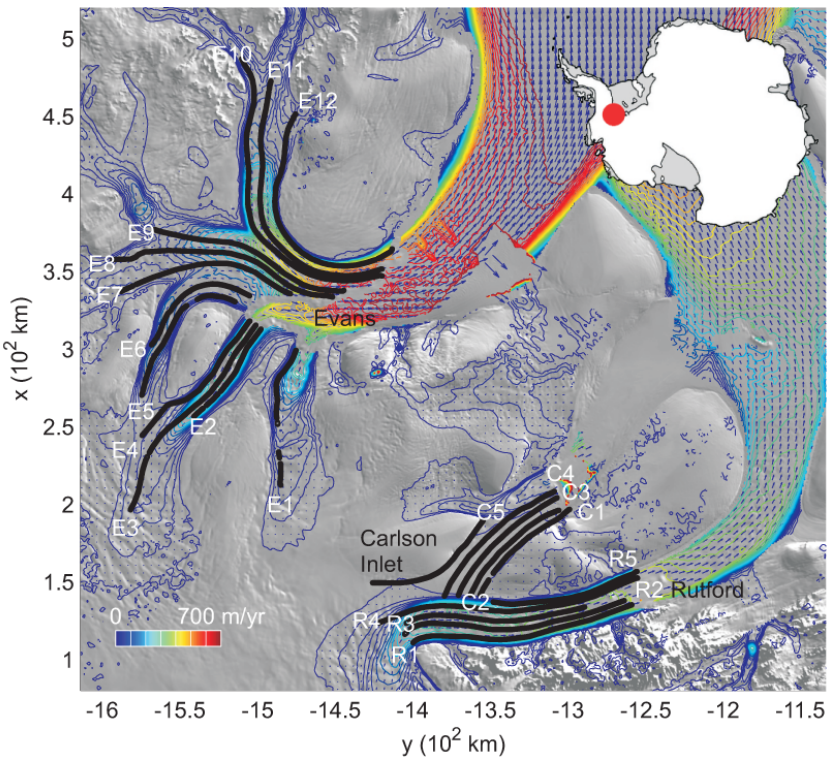

Fig. 2. Map showing the Rutford Ice Stream, Evans Ice Stream and Carlson Inlet which flow into the Filchner-Ronne Ice Shelf (FRIS), West Antarctica (see inset). The colour scale and arrows indicate the InSAR-derived surface velocity field (Rignot et al., 2011) overlaid on a subset of the MODIS mosaic for Antarctica. Only velocities higher than $20 \mathrm{~m} \mathrm{yr}^{-1}$ are shown. Black lines represent the $22 \mathrm{sec}-$ tions of radar profiles that were analysed in this work.

More details about the radar system and data processing methods can be found in Corr et al. (2007), Vaughan et al. (2006) and Rippin et al. (2011). Comparison of ice thickness recorded at flight-line crossover points for a similar RES survey using the same equipment at Pine Island Glacier has indicated a root-mean-square error of $\sim 23 \mathrm{~m}$ (Vaughan et al., 2006). The errors from crossovers in measurements of icesurface elevation were considerably lower, $\sim 1 \mathrm{~m}$. The greatest source of uncertainty for the surface measurements is the false determination of the airplane's position; the errors in the ice thickness result largely from off-nadir reflections and interpretation/digitizing uncertainty. The errors in surface and bed profiles are small, and they are not of any concern for our work.

For our analysis, we selected 17 segments of flight lines along fast-flowing sections of the ice sheet. They are represented by the black lines in Fig. 2, and enumerated R1-R5 for the Rutford Ice Stream and E1-E12 for the Evans Ice Stream. Moreover, 5 profiles selected for the slow-moving Carlson Inlet are indicated by $\mathrm{C} 1-\mathrm{C} 5$ in Fig. 2. The surface and bed profiles for all flight lines are shown in Fig. 3. All 22 sections satisfy the following criteria:

1. Each radar line was flown approximately parallel to the ice flow direction. This allowed us to treat the bed-tosurface transfer problem in a two-dimensional plane (1 horizontal and 1 vertical direction), with the ice velocity vector lying in that plane. Transverse effects cannot be excluded, but in Sect. 5 we show that they do not influence the qualitative aspects of the relationship between transfer amplitude and $\lambda_{\mathrm{x}} / H$.

2. All profiles were restricted to the grounded part of the ice sheet. Some sections were cut off at their upstream end to reduce variations in flow conditions, i.e. variations in surface and basal velocity. For all profiles along the Rutford and Evans ice streams, velocities vary from $50 \mathrm{~m} \mathrm{yr}^{-1}$ or more at the upstream end to several hundred meters per year near the grounding line. Despite the large variability in flow speed along each profile, there is a distinctive difference with profiles along the Carlson Inlet, which have velocities of typically a few tens of meters per year. In addition, profile lengths needed to be sufficiently long, as we are interested in elevation perturbations with wavelengths up to 20 times the ice thickness. For our dataset, track lengths (l) vary from $l=72 \mathrm{~km}$ (E2) to $l=207 \mathrm{~km}(\mathrm{E} 10)$, and the mean ice thickness along profiles has values between $H=1316 \mathrm{~m}$ (E12) and $H=2631 \mathrm{~m}$ (E3).

3. There are no substantial gaps in the data, although some smaller gaps $(<5 \mathrm{~km})$ are present for R2, C1, E2, E3 and E7. Two profiles, E1 and E5, with larger gaps of $18 \mathrm{~km}$ and $8 \mathrm{~km}$, respectively were retained, and results for these two profiles should thus be treated with greater caution.

\subsection{Plane-slab approximation}

For each flow-line the surface and bed profiles ( $s$ and $b$ ) were separated into a mean value and a deviatoric part, following Eq. (2). The mean values were obtained from a linear regression of the elevation data and were used to determine the parameters of a plane-slab plug flow: the mean slope $\alpha$ was set equal to the slope of the surface trend $s^{(0)}$, and the mean ice thickness $H$ was chosen as the difference between $s^{(0)}$ and $b^{(0)}$ averaged along the profile. The results are shown as dashed blue lines in Fig. 3. The mean amplitude of the deviations $\Delta s$ and $\Delta b$ is smaller than $0.01 H$ and $0.25 H$, respectively, so a comparison of our results to perturbation theory is justified.

The mean slipperiness or slip ratio along each profile was determined from estimates of the mean forward deformational velocity and mean basal sliding velocity, according to Eq. (3). The mean basal sliding velocity is given by the difference between the mean surface velocity and the forward deformation, where the former was estimated from 1-km resolution InSAR data (Rignot et al., 2011). The forward deformational velocity was computed from $v_{d, x}^{(0)}=\frac{1}{2} A[\rho g \sin (\alpha)]^{3} H^{4}$, where we chose $n=3$ and a spatially constant rate factor for ice at $-10^{\circ} \mathrm{C}$, i.e. $A=3.5 \times$ $10^{-25} \mathrm{~s}^{-1} \mathrm{~Pa}^{-3}$ (Cuffey and Paterson, 2010). Although the ice viscosity is generally a function of depth and position 


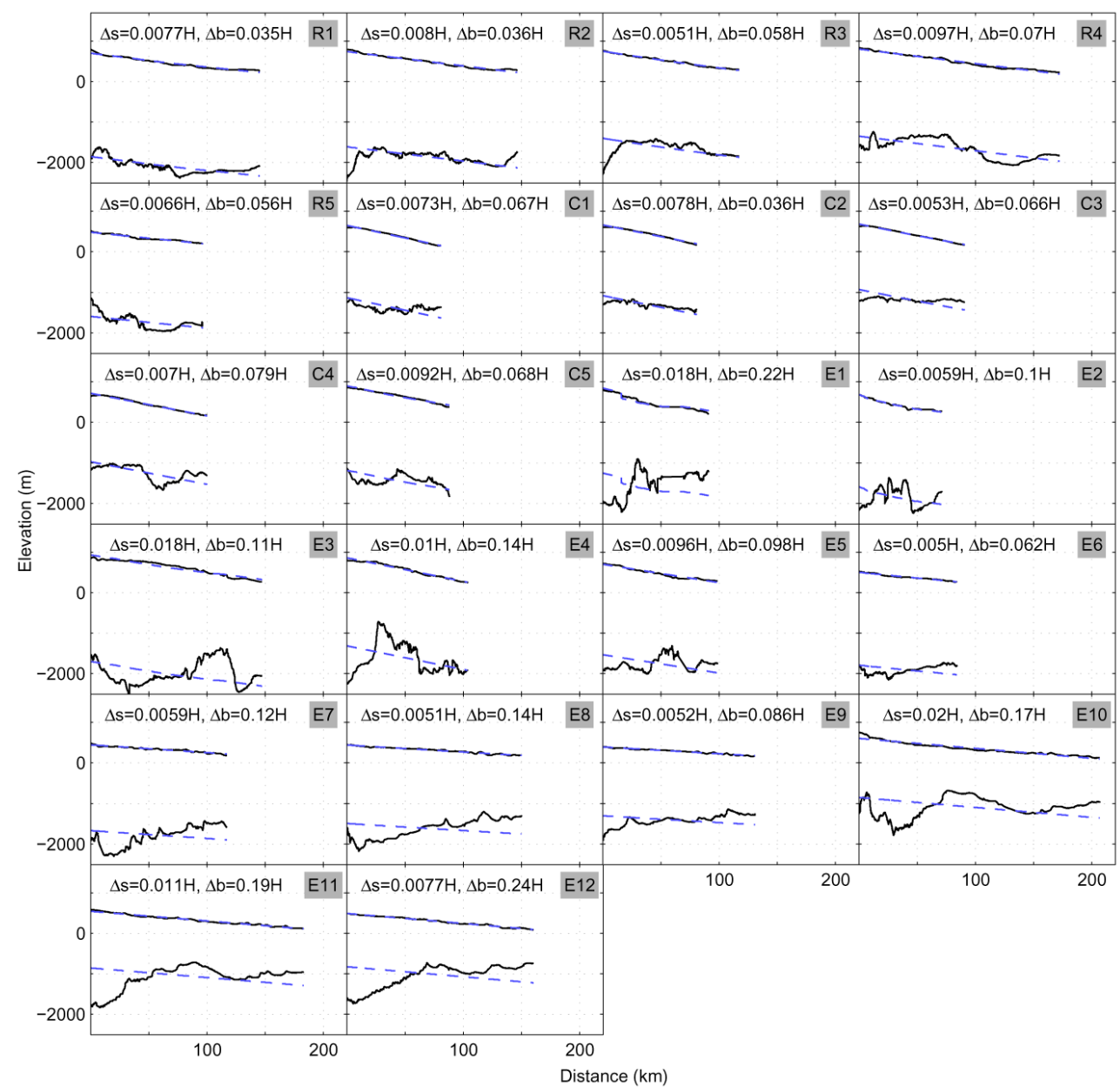

Fig. 3. Black lines show the surface and bed returns along the radar profiles mapped in Fig. 2; dashed blue lines represent the plane-slab approximation used in this work; and $\Delta s$ and $\Delta b$ are the mean amplitude of the surface and bed deviations. More details can be found in Sect. 3.2.

along the flow, exact knowledge about $A$ is not required as it only enters our analysis via the mean slip ratio. The latter is inversely proportional to $A$, which is a weak dependency in the context of this work, as can be illustrated for a typical active ice stream with a mean surface velocity of $250 \mathrm{~m} \mathrm{a}^{-1}$, a mean surface slope of $0.003 \mathrm{rad}$ and a mean ice thickness of $1500 \mathrm{~m}$. Assuming an upper and a lower limit for $A$ corresponding to temperate ice and polar ice at $-20^{\circ} \mathrm{C}$, respectively, values for the slip ratio vary from $C^{(0)} \approx 60$ to $C^{(0)} \approx 1200$. Even for these extreme (and unrealistic) ice properties, the slip ratio is significantly larger than unity, and a peak in the transfer function is expected.

In summary, values for both $v_{b, x}^{(0)}$ and $v_{d, x}^{(0)}$ were obtained for each profile, resulting in estimates for the mean slip ratio ranging from $C^{(0)}<1$ for flight lines along the slow-flowing Carlson Inlet to $C^{(0)}>1000$ for the fast-flowing central sections of the Evans Ice Stream (see Fig. 4). As emphasized above, the conclusions of our analysis are insensitive to the exact value of $C^{(0)}$, since theory predicts a local peak in the bed-to-surface transfer amplitude as long as $C^{(0)} \gg 1$. For completeness, the extreme values of $C^{(0)}$ corresponding to temperate ice (lower limit) and polar ice at $-20^{\circ} \mathrm{C}$ (upper limit) are added as error bars in Fig 5.

\subsection{Estimated transfer amplitudes}

For each flight line we estimated the along-flow transfer amplitude between the basal and surface undulations $(\Delta b$ and $\Delta s$ ) as a function of wavelength. The estimated amplitudes will be denoted by $T_{\mathrm{sb}}^{(\mathrm{obs})}\left(\lambda_{\mathrm{x}}\right)$ and are defined as the quotient of the cross power spectral density of $\Delta b$ and $\Delta s$ (i.e. the Fourier transform of the cross-correlation between the bed and surface signal) and the power spectral density of $\Delta b$ (i.e. the Fourier transform of the autocorrelation of the bed signal). 


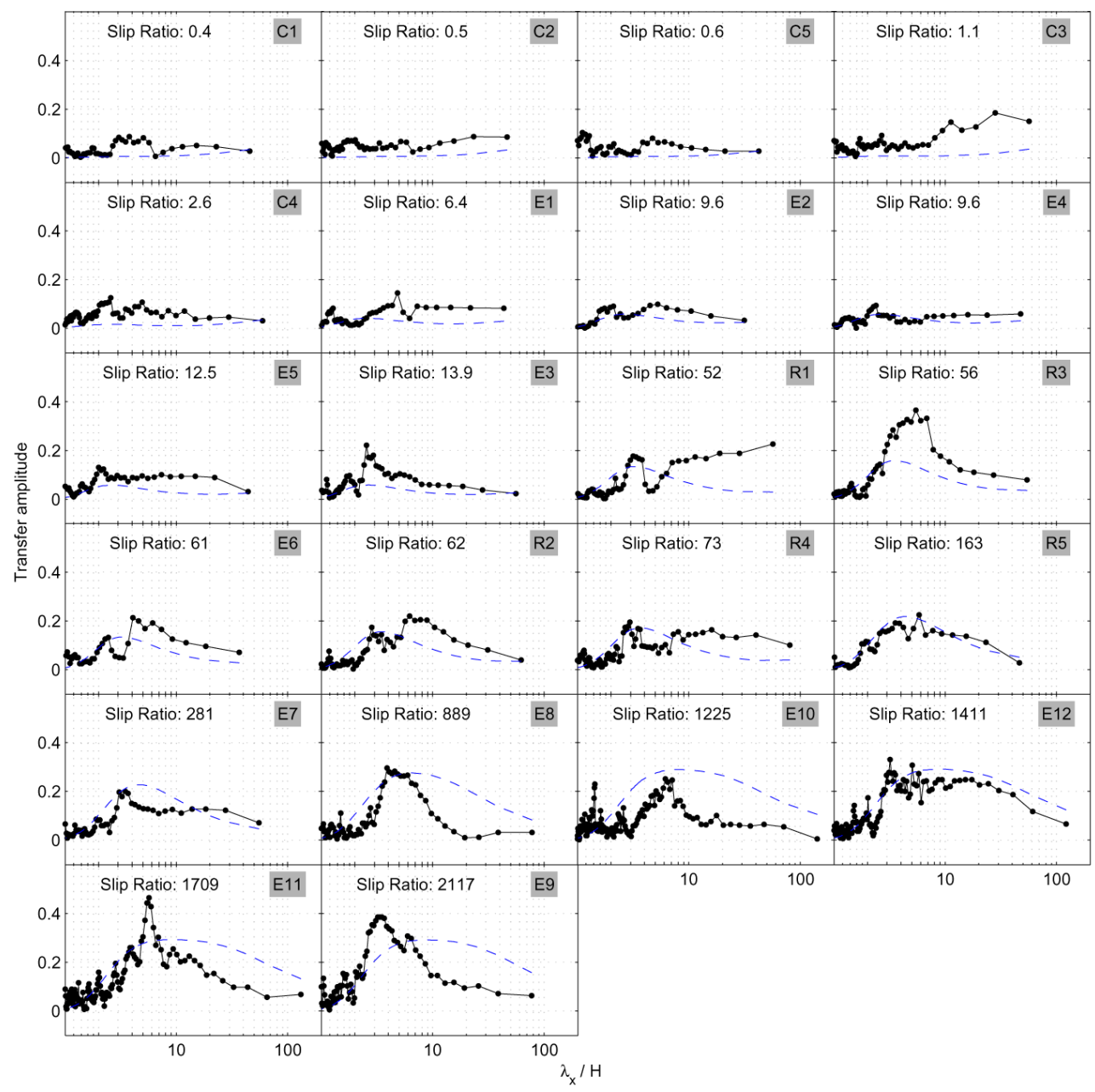

Fig. 4. Estimated transfer amplitudes for radar profiles along the Carlson Inlet (C1-C5), Rutford Ice Stream (R1-R5) and Evans Ice Stream (E1-E12), ranked from low to high values of the slip ratio. The dashed blue lines show the corresponding flow-line prediction, scaled by a factor $f=0.3$ in order to account for three-dimensional effects. The value of $f$ was obtained from an optimal least-square fit with the observations.

The evaluation of $T_{\mathrm{sb}}^{(\mathrm{obs})}$ requires a Fourier-based analysis of the data. Therefore, each radar section was re-sampled onto an equally spaced grid of $2^{N}$ data points. The value of $N$ was chosen independently for each profile, such that the new sampling interval is close to the mean original sampling interval (between $19.9 \mathrm{~m}$ and $23.8 \mathrm{~m}$ ). The new data points were obtained using a cubic spline interpolation method, which also fills the data gaps. It should be noted that our results do not crucially depend on this interpolation method, and a piecewise linear interpolation could be used instead.

The calculation of transfer amplitudes (or equivalently, power spectra) from observational data can be surprisingly difficult, and results are often sensitive to various assumptions made in the analysis method. A common issue is the leakage of power between adjacent frequency bands, which results in a large variance for the spectral coefficients. In order to minimize this systematic error, we used Thompson's multitaper spectrum estimation method (Thomson, 1982; Prieto et al., 2009) with two Slepian tapers and a time bandwidth product of 4 . Results were cross-checked using Welch's averaged periodogram method (Welch, 1967; Press et al., 2007) with a Hamming window applied to a minimum of two overlapping segments with a fixed length of $45 \mathrm{~km}$. Although the quantitative results were found to be sensitive to specific parameters in the applied processing (such as the number of tapers or the length of the Hamming window), qualitative results were robust and independent of the chosen method. In this text we only present results from the multitaper analysis.

\section{Results}

The estimated transfer amplitudes for all profiles are shown in Fig. 4, ranked from low values of the mean slip ratio in 


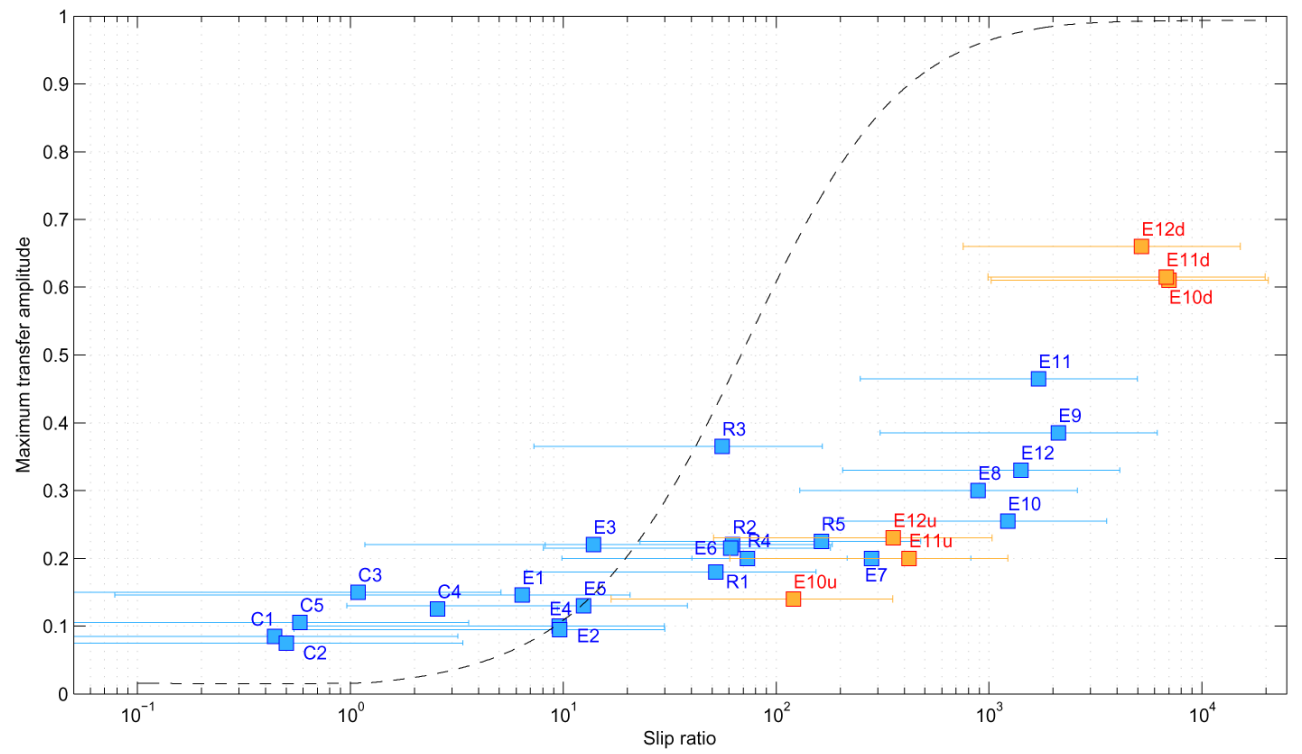

Fig. 5. Blue squares represent a scatter plot of the maximum transfer amplitude as a function of the mean slip ratio for all 22 profiles in this study. The plot shows a clear increasing trend for increasing values of the slip ratio. Error bars depict the maximum uncertainty in the slip ratio due to a lack of knowledge about the ice viscosity. Lower limits correspond to the assumption of a temperate ice column, whereas upper limits are obtained for a uniform ice temperature of $-20^{\circ} \mathrm{C}$. The dashed line corresponds to the flow-line prediction of the full Stokes system for $\alpha=0.003 \mathrm{rad}$. Orange data points show the maximum transfer amplitude and mean slip ratio for the upper sections (denoted by an index $u$ ) and lower sections (denoted by an index $d$ ) along flight lines E10, E11 and E12.

the top left to high values in the bottom right. Additionally, the dashed blue line in each figure represents the theoretical flow-line approximation, $T_{\mathrm{sb}}\left(\lambda_{\mathrm{x}}, C^{(0)}, \alpha, H\right)$, with $C^{(0)}$ and $\alpha$ the estimated slip ratio and mean surface slope, respectively. As will be detailed in Sect. ??, the flow-line approximation generally overestimates the transfer amplitude as it neglects transverse basal perturbations which generally dampen the surface response and which cannot be accounted for from available observational data. In order to incorporate possible three-dimensional effects, all flow-line curves in Fig. 4 have been rescaled by a constant factor $f=0.3$, which was obtained from an optimal least square fit with observations. Although the physical origin of the scaling factor $f<1$ is clear, the accuracy of the fit is rather poor in some cases (e.g. R3 and E10) and much better in other cases (e.g. R5 and E12). This variability can be related to uncertainties in our estimate of the mean slip ratio $C^{(0)}$, but it also suggests that the value of $f$ is not unique across different profiles or across the entire range of wavelengths. Hence, three-dimensional effects cannot be captured by a simple parametrization. In addition, it should be noted that the obtained scaling factor depends on particular details of the multitaper analysis method, which therefore reduces the physical relevance of its value.

The results depicted in Fig. 4 can be divided into three broad categories, depending on the value of the slip ratio. Profiles $\mathrm{C} 1-\mathrm{C} 5$ run along a steeper and slow-flowing section of the ice sheet, which results in larger internal deformation and low values of the slip ratio: $0.4 \leq C^{(0)} \leq 2.6$. For all these profiles, the theoretical estimate for the transfer amplitude is close to zero with a slight increase in transfer towards longer wavelengths. The observed amplitudes roughly follow this pattern, although the results lie systematically above the theoretical prediction and an increase towards longer wavelengths is not generally observed. Only in the case of $\mathrm{C} 2$ and $\mathrm{C} 3$, the transfer amplitude reaches a maximum at wavelengths above 10 ice thicknesses, whereas other profiles are relatively constant.

The second set of profiles, E1-E5, have mean slip ratios which are an order of magnitude larger. These sections follow relatively steep surface slopes with mean ice thickness values that vary from $2100 \mathrm{~m}$ to $2600 \mathrm{~m}$. For these profiles the internal deformation velocity contributes to up to $15 \%$ of the surface speed, which leads to estimates of the mean slip ratio with values between 6.4 and 13.9. The theoretical curves in Fig. 4 predict a slight increase in transfer for wavelengths between 1 and 10 ice thicknesses, whereas the observed transfer amplitudes show a more varied behaviour: E2 and E4 follow the theoretical result, whereas E1 and E5 overestimate the transfer at wavelengths above 10 times the mean ice thickness, and E3 shows a distinctive maximum for wavelengths between 2 and 3 ice thicknesses. Except for the latter case, none of the other profiles shows a noticeable increase of transfer at intermediate wavelengths.

The remaining profiles, E6-E12 and R1-R5, have mean slip ratios ranging from 52 to 2111, which is reflected in higher surface speeds and/or less internal deformation. As 
expected from theory, all observed transfer amplitudes have a distinctive maximum at wavelengths between 1 and 20 ice thicknesses, providing strong evidence for the efficient transfer of medium-scale bedrock undulations to the surface of fast-flowing sections of the Rutford and Evans ice streams.

The results in Fig. 4 express a clear dependence of the peak amplitude on the mean slip ratio. This relationship is further exhibited in Fig. 5, which shows a scatter plot of slip ratios versus peak amplitudes for all profiles (blue squares). The estimated peak amplitudes are defined as the maximum of each transfer function between 1 and 20 ice thicknesses. The error bars in Fig. 5 correspond to extreme values of the slip ratio for each profile; the lower limit corresponds to its value for temperate ice, whereas the upper limit is computed for a uniform ice column at $-20^{\circ} \mathrm{C}$. Despite a substantial amount of scatter, the plot indicates a 3.5-fold increase over the considered range of slip ratios. This result is supported by theory (dashed line in Fig. 5), although the flow-line model predicts a more rapid increase and a peak amplitude which converges quickly towards unity for mean slip ratios between 10 and 1000 .

In all of the analysis so far, we have assumed a constant value for the slip ratio along each profile, with the error bars in Fig. 5 indicating a maximum range of uncertainty due to unknown variations in the ice viscosity. In addition, it is feasible to assume that along-flow values of $C^{(0)}$ can change considerably due to variations in surface slope and surface speed. As a result, the transfer characteristics of an ice stream are spatially variable, with a gradual increase of the transfer from the upstream catchment towards the fast-flowing region near the grounding line. For longer flight lines this variability can be investigated by splitting the radar profiles into different sections and by analysing these sections separately. Results of such an analysis for the three longest profiles, E10 $(207 \mathrm{~km}), \mathrm{E} 11(183 \mathrm{~km})$ and E12 $(160 \mathrm{~km})$, are represented by the red datapoints in Fig 5. It is clear that, for the upper sections (denoted by E10u, E11u and E12u), the mean slip ratio is suppressed due to lower ice velocities, and the transfer has decreased simultaneously. On the other hand, for the fast-flowing downstream sections (denoted by E10d, E11d and E12d), the mean slip ratio is significantly higher and the observed transfer is enhanced accordingly. These findings underline the large variability in the slip ratio and transfer along long profiles, but E10d, E11d and E12d also provide strong additional evidence for the efficient transfer of small bedrock perturbations to the surface of fast-flowing sections of an ice stream.

In summary, the data confirm the existence of a maximum transfer amplitude for $C^{(0)} \gg 1$, and validate theoretical predictions about the relation between $C^{(0)}$ and the maximum transfer amplitude. Despite this good qualitative agreement between theory and observations, a more detailed comparison also marks several discrepancies. First, it should be noted that, unlike for theoretical estimates, most observed amplitudes do not have one continuous peak, but consist of several local maxima. A separation into two or more peaks, not necessarily of equal height, is apparent for most profiles, with R3 and E7-E8 being exceptions. Another notable difference is related to the absolute value of the peak height. Recall that the theoretical curves in Fig. 4 are rescaled by a factor 0.3, and hence observed peak amplitudes are generally lower than expected from theoretical flow-line estimates. In general, the transfer is dampened by at least $60 \%$ and up to $70 \%$, except for C1-C5 for which all estimates systematically exceed theoretical values. This dampening is also clear from Fig. 5, which shows observed peak heights as a function of the slip ratio and where the dashed line represents the theoretical prediction. For $C^{(0)}>20$, observed peak amplitudes lie consistently below the theoretical curve. The potential physical and/or methodical origins of these discrepancies are numerous, and it is arguable that a complicated combination of several effects influences the observations. We will restrict our discussion to an overview of the principle mechanisms that might affect the results, and we provide a list of theoretical assumptions that need to be considered in order to aim for a better fit with observations.

\section{Discrepancies between flow-line theory and observations}

For all profiles with a slip ratio $C^{(0)}>20$, the observed transfer amplitudes are consistently lower than the full Stokes flow-line predictions. Such a suppression of the surface response can have several (indistinguishable) causes.

Three-dimensional effects: the most significant source of transfer dampening is related to the inherent threedimensional character of the problem at hand. In the foregoing analysis, we have assumed that all analysed radar profiles were, to good approximation, parallel to the surface velocity field, and that transverse ice dynamical effects could be neglected. These assumptions were implemented in the theoretical transfer amplitude by taking the flow-line limit $\lambda_{y} \rightarrow \infty$ of the full three-dimensional solution $T_{\mathrm{sb}}\left(\lambda_{\mathrm{x}}, \lambda_{\mathrm{y}}, C^{(0)}, \alpha\right)$. In reality, corrections to the flow-line model come from a combination of different sources: (i) flight lines are likely to have a small and variable across-flow component; (ii) the limit $\lambda_{y} \rightarrow \infty$ is not exact as bedrock features have a finite size in the transverse direction; and (iii) the ice streams in this study are confined by lateral margins. Due to the lack of observational knowledge about transverse bedrock and surface properties, an assessment of three-dimensional effects relies on synthetic modelling. 

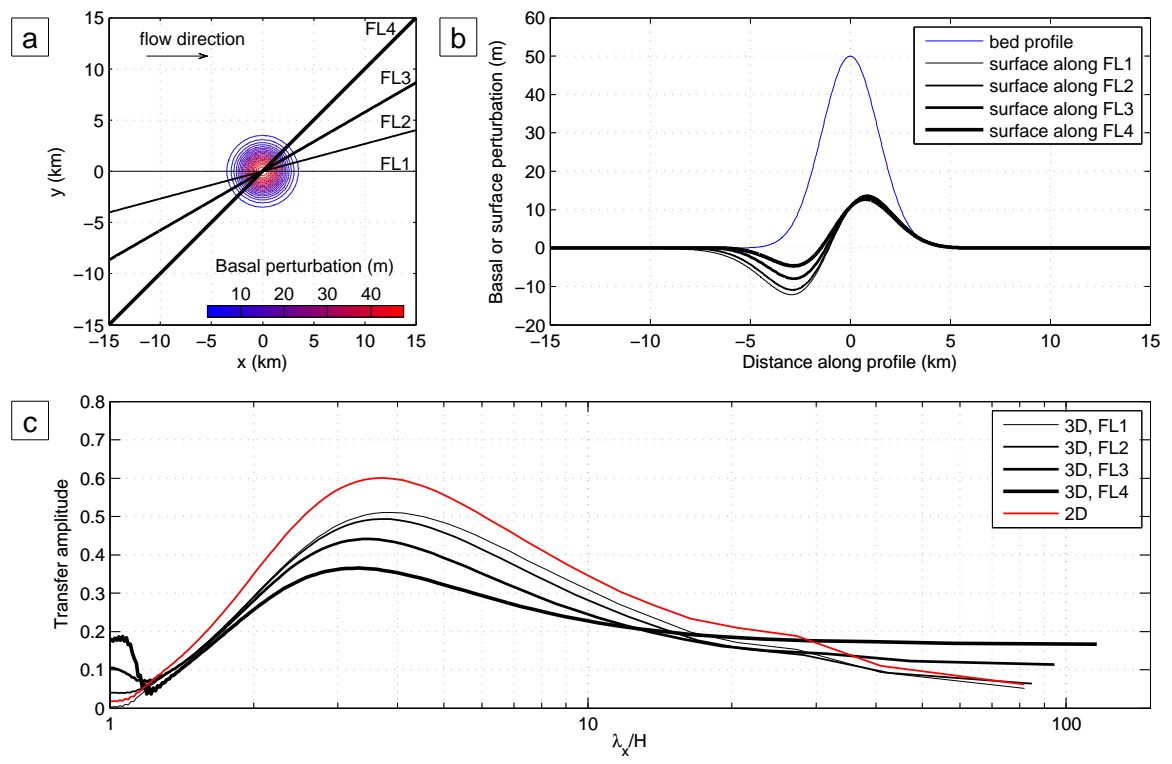

Fig. 6. (a) Gaussian-shaped bedrock undulation which was used to simulate the three-dimensional flow. Sections were taken at an angle of $0,15,30$ and 45 degrees with respect to the flow direction and are denoted by FL1, FL2, FL3 and FL4, respectively. (b) The blue curve represents the basal perturbation which is identical along each section. Black curves represent the three-dimensional surface response along each section, showing a clear angle dependence with increased dampening of the transfer for larger angles. (c) Transfer amplitudes estimated from $\Delta s$ and $\Delta b$ along each section (black lines), as well as the two-dimensional result (red curve) for the same bedrock profile, uniform inclination and slip ratio. The two-dimensional flow-line limit neglects three-dimensional effects, which leads to a systematic overestimation of the transfer at wavelengths less than 20 times the mean ice thickness.

Previous modelling efforts by Reeh (1987) and Sergienko (2012) have shown that a non-zero ratio between longitudinal and transverse wavelengths can have a substantial effect on the flow as the wavelength ratio increases, and surface amplitudes can be reduced by more than one order of magnitude as compared to the two-dimensional plane approximation. In order to study the effect of finite transverse wavelengths on the estimated transfer amplitude, we analyse a full Stokes flow with linear ice rheology over a Gaussianshaped bedrock undulation with mean bedrock inclination $\alpha=0.003 \mathrm{rad}$ and mean slipperiness $C^{(0)}=100$. Figures $6 \mathrm{a}$ and $6 \mathrm{~b}$ show the bedrock profile and surface response along four different transects (denoted by FL1-FL4) which are oriented at different angles with respect to the flow direction. The bed-to-surface transfer for each profile is presented in Fig. $6 c$ (black curves), together with the two-dimensional flow-line prediction (red curve). The results show two clear tendencies: (i) the three-dimensional transfer in the direction of the flow (FL1) is suppressed by about $15 \%$ as compared to two-dimensional result, and (ii) the suppression of the transfer increases as the angle between the flight line and the flow direction increases. This systematic suppression of the transfer amplitude caused by transverse effects has the potential to explain why observed transfer amplitudes are consistently smaller than expected from flow-line theory.

Non-isothermal ice: all theoretical results discussed so far were derived for a spatially constant viscosity, whereas more realistic treatments use a depth-dependent relation for $\eta$. For example, if one assumes an exponential decrease of the viscosity with depth,

$\eta(z)=\eta_{\mathrm{s}} \mathrm{e}^{\mu z}$,

with $\eta_{\mathrm{s}}$ the viscosity at the surface, and $\mu$ a factor that scales the vertical variation of $\eta$, then the full Stokes system can be solved analytically, and one finds that a gradual increase of the viscosity towards the surface leads to a systematic reduction of the transfer (Gudmundsson, 2004b). The amount of reduction depends on $\mu$, a parameter that can vary along an ice stream and between different ice streams. The reason for the reduced transfer is the presence of stiffer, more viscous ice near the surface, which has a dampening effect. We have partially taken this effect into account by setting $\eta$ equal to its recommended base value at $-10^{\circ} \mathrm{C}$. As such, the ice stream is neither treated as a temperate feature $\left(\sim 0^{\circ} \mathrm{C}\right)$ with a value for $\eta$ that is generally too low, nor is it treated as a cold feature $\left(\simeq 20^{\circ} \mathrm{C}\right)$ with a value for $\eta$ that is too high and only valid in the uppermost layers.

Ice rheology: analytical solutions for the transfer in the full Stokes system are only known for a linear flow law and linear sliding law. It has been pointed out by Raymond and Gudmundsson (2005) that higher values of Glen's exponent generally lead to an increase of the maximum transfer by about $20 \%$ for $n=3$. On the other hand, the effect of a non-linear sliding law on the maximum transfer amplitude is negligi- 
ble. As it is generally assumed that the physical value of $n$ is larger than unity, this result at least partially offsets the dampening effects discussed above. Also anisotropic properties of the ice affect the flow and hence the transfer characteristics. In the simplest cases, anisotropic effects are conveniently parametrized by a constant enhancement factor in Glen's flow law, which effectively describes a softening of the ice and therefore an increase in the transfer. The uncertainties related to this effect are included in the error bars in Fig. 5, which correspond to extreme limits for the ice viscosity.

\section{Conclusions}

We have analysed a comprehensive set of radar profiles along fast- and slow-flowing sections of the West Antarctic ice sheet and determined the response of surface elevations to variations in the bed topography. Our analysis shows that an accurate estimate of the transfer amplitude for wavelengths up to 20 ice thicknesses requires radar profiles of at least $70 \mathrm{~km}$ in length. Since such datasets have only recently become available, our study provides the first empirical test for theoretical results on transfer amplitudes.

We find that for large values of the slip ratio, i.e. for fastflowing ice streams, the surface topography carries important information about bedrock undulations with wavelengths between 1 and 20 times the mean ice thickness. The efficiency of the bed-to-surface transfer at these wavelengths increases by a factor of $\sim 3.5$ as slip ratios increase from $\sim 1$ to several thousand. These observations are in good qualitative agreement with the theoretical results obtained for a full Stokes system by Gudmundsson (2003) and Raymond and Gudmundsson (2005). Results from the slow-flowing Carlson Inlet confirm the important difference between the flow characteristics of ice streams for which most ( $95 \%$ and often more) of the velocity is due to basal sliding, and alpine-type glaciers which attain a considerable fraction of their surface speed by internal creep. The latter do not exhibit any significant transfer of small-scale topographic features to the surface.

Several differences between observations and theoretical flow-line predictions have been observed and clarified, although some discrepancies remain unexplained. A leastsquare fit to flow-line theory shows that the measured transfer for fast-flowing ice is systematically reduced by a factor of $70 \%$ as compared to the flow-line prediction, which is arguably due to the inherent three-dimensional character of an ice stream. For instance, the presence of finite transverse bedrock undulations leads to a reduction in the surface expression of longitudinal wavelengths. Additionally, nonisothermal ice properties generate a layer of stiffer ice near the surface, which dampens the transfer.
Our analysis provides an important verification for the use of current theoretical models in ongoing efforts to study ice stream dynamics, and for their use in inverse methods to obtain information about basal perturbations (topography and slipperiness) from surface measurements. Results show a good qualitative agreement with the full Stokes theory, which suggests that other predictions of this theory can also be trusted and are of importance for our understanding of ice stream dynamics. For example, Gudmundsson (2003) shows that the length scale which characterizes the transmission of horizontal deviatoric stresses increases with increasing values of the slip ratio. This length scale becomes particularly large (100 or 1000 times the mean ice thickness) for fast-flowing ice streams, which means that its value is controlled by ice-stream width rather than ice thickness. On the other hand, results of our analysis are not captured by models that are based on the shallow ice sheet and shallow ice stream approximations. These approximations, which are designed to study intermediate to long wavelength problems, either grossly overestimate the transfer at wavelengths $<10 H$ (shallow ice stream) or do not reproduce an increase in the transfer at intermediate wavelengths at all (shallow ice sheet), as can be seen from Fig. 1. As such, forward (inversion) results about the surface (basal) topography at these wavelengths are bound to be erroneous when obtained from shallow ice flow models commonly used for the inversion of basal properties.

Finally, we stress that the correspondence between observed and theoretical transfer amplitudes implies that local surface undulations of the Rutford and Evans ice streams are largely controlled by local variation in the bedrock topography. In our analysis, which is restricted to wavelengths less than 20 times the mean ice thickness, we find no direct evidence for the impact of variations in basal slipperiness on surface topography. Theoretical results indeed suggest that the effect of small-scale perturbations in slipperiness only becomes relevant at wavelengths much larger than 20 times the mean ice thickness (Gudmundsson, 2003). It also confirms previous results for the Rutford Ice Stream (Raymond-Pralong and Gudmundsson, 2011), and suggests that variations in basal topography should be treated carefully when inverting surface data for spatial variation in basal shear stress at length scales less than 20 ice thicknesses.

Acknowledgements. Richard Hindmarch and Rob Bingham designed the flight lines for the radar survey, and Ian Joughin supplied the surface velocity data. We are grateful for helpful suggestions from the editor Olivier Gagliardini, and Rupert Gladstone and two anonymous reviewers.

Edited by: O. Gagliardini 


\section{References}

Arthern, R. J. and Gudmundsson, G. H.. Initialization of ice-sheet forecasts viewed as an inverse Robin problem, J. Glaciol., 560, 527-533, 2010.

Bamber, J. L.,Vaughan, D. G. , and Joughin, I.: Widespread complex flow in the interior of the Antarctic ice sheet, Science, 2870, 1248-1250, 2000.

Budd, W. F.: Ice flow over bedrock perturbations, J. Glaciol., 90, 29-48, 1970.

Corr, H. F. J., Ferraccioli, F., Frearson, N., Jordan, T., Robinson, C., Armadillo, E., Caneva, G., Bozzo, E., and Tabacco, I.: Airborne radio-echo sounding of the Wilkes Subglacial Basin, the Transantarctic Mountains, and the Dome $\mathrm{C}$ region, Terra Ant. Reports, 13, 55-63, 2007.

Cuffey, K. M. and Paterson, W. S. B.: The Physics of Glaciers, 4th Edn., Elsevier, Burlington, USA, 2010.

Gudmundsson, G. H.: Transmission of basal variability to a glacier surface, J. Geophys. Res., 1080, 1-19, 2003.

Gudmundsson, G. H.: Estimating basal properties of glaciers from surface measurements, in: Glaciers and earth's changing environment, Blackwell Publishing Ltd., Oxford, UK, 2004a.

Gudmundsson, G. H.: The effect of non-isothermal ice on the transfer of bed properties to the surface of fast-flowing ice streams, unpublished, 2004b.

Gudmundsson, G. H.: Analytical solutions for the surface response to small amplitude perturbations in boundary data in the shallow-ice-stream approximation, The Cryosphere, 2, 77-93, doi:10.5194/tc-2-77-2008, 2008.

Hutter, K.: Theoretical Glaciology; Material Science of Ice and the Mechanics of Glaciers and Ice Sheets, D. Reidel, Norwell, Mass., 1983.

Johannesson, T.: Landscape of temperate ice caps, $\mathrm{PhD}$ thesis, University of Washington, Seattle, USA, 1992.

Joughin, I., MacAyeal, D. R., and Tulaczyk, S.:. Basal shear stress of the Ross ice streams from control method inversions, J. Geophys. Res.-Sol. Earth, 20 pp., 2004.

Joughin, I. , Bamber, J. L.,Scambos, T. ,Tulaczyk, S. ,Fahnestock, M. , and MacAyeal, D. R.: Integrating satellite observations with modelling: basal shear stress of the Filcher-Ronne ice streams, Antarctica, Philos. T. R. Soc. A, 3640, 1795-814, 2006.

Landon, J. and Raymond, C. F.: Chislenniy raschet reaktsii poverkhnosti lednika na izmeneniya tolshchiny l'da [Numerical calculation of adjustment of a glacier surface to pertubations of ice thickness], Mater. Glyatsiol. Issled. Khron. Obsuzhdeniya, 32, 233-239, 1978.
MacAyeal, D. R., Bindschadler, R. A., and Scambos, T. A.: Basal friction of Ice Stream E, West Antarctica, J. Glaciol., 410, 247 262, 1995.

Nye, J. F. The motion of ice sheets and glaciers, J. Glaciol., 30, 493-507, 1959.

Press, W. H., Teukolsky, S. A., Vetterling, W. T., and Flannery, B. P.: Numerical Recipes 3rd Edition: The Art of Scientific Computing, Cambridge University Press, Cambridge, UK, 2007.

Prieto, G. A., Parker, R. L., and Vernon III, F. L.: A Fortran 90 library for multitaper spectrum analysis. Computers and Geosciences, 350, 1701-1710, 2009.

Raymond, M. J. and Gudmundsson, G. H.: On the relationship between surface and basal properties on glaciers, ice sheets, and ice streams, J. Geophys. Res., 1100, 1-17, 2005.

Raymond, M. J. and Gudmundsson, G. H.: Estimating basal properties of ice streams from surface measurements: a nonlinear Bayesian inverse approach applied to synthetic data, The Cryosphere, 3, 265-278, doi:10.5194/tc-3-265-2009, 2009.

Raymond-Pralong, M. and Gudmundsson, G. H.: Bayesian estimation of basal conditions on Rutford Ice Stream, West Antarctica, from surface data, J. Glaciol., 570, 315-324, 2011.

Reeh, N.: Steady-state three-dimensional ice flow over an undulating base: first-order theory with linear ice rheology, J. Glaciol., 330, 177-185, 1987.

Rignot, E., Mouginot, J., and Scheuchl, B.: Antarctic grounding line mapping from differential satellite radar interferometry, Geophys. Res. Lett., 380, 1-6, 2011.

Rippin, D. M., Vaughan, D. G., and Corr, H. F. J.: The basal roughness of Pine Island Glacier, West Antarctica, J. Glaciol., 570, 6776, 2011.

Sergienko, O. V.: The effects of transverse bed topography variations in ice-flow models, J. Geophys. Res., 117, 1-16, 2012.

Thomson, D. J.: Spectrum estimation and harmonic analysis, Proceedings of the IEEE, 700, 1055-1096, 1982.

Vaughan, D. G., Corr, H. F. J., Ferraccioli, F., Frearson, N., O’Hare, A., Mach, D., Holt, J. W., Blankenship, D. D., Morse, D. L., and Young, D. A.: New boundary conditions for the West Antarctic ice sheet: Subglacial topography beneath Pine Island Glacier, Geophys. Res. Lett., 330, 2-5, 2006.

Vieli, A. and Payne, A. J.: Application of control methods for modelling the flow of Pine Island Glacier, West Antarctica, Ann. Glaciol., 360, 197-204, 2003.

Welch, P.: The use of fast Fourier transform for the estimation of power spectra: A method based on time averaging over short, modified periodograms, IEEE Trans. Audio Electr., 150, 70-73, 1967. 